\title{
The abscopal effect: efficacy of radiotherapy in patients on progression after treatment with ipilimumab $3 \mathrm{mg} / \mathrm{kg}$
}

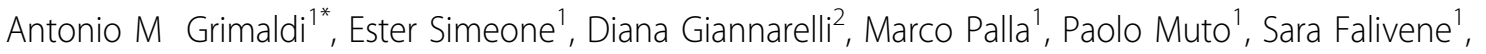

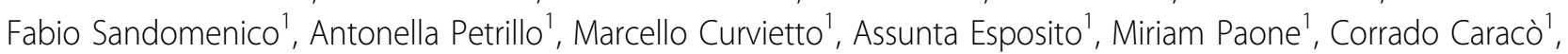 \\ Gennaro Ciliberto ${ }^{1}$, Nicola Mozzillo ${ }^{1}$, Paolo A Ascierto ${ }^{1}$
}

From Society for Immunotherapy of Cancer 28th Annual Meeting

National Harbor, MD, USA. 8-10 November 2013

\section{Background}

After more than 30 years, Ipilimumab was the first agent which showed a survival benefit for the treatment of metastatic melanoma. However, only about the $20 \%$ of patients have a long-term survival benefit. The combination of ipilimumab with other therapies might improve its efficacy.

A new phenomenon also known as abscopal effect refers to a regression of metastatic lesions distant from the primary site of radiotherapy (RT). This systemic response is observed in patients who received ipilimumab.

Here we reported the outcomes from patients treated in the ipilimumab Italian expanded access program (EAP) who received $\mathrm{RT}$ after ipilimumab progression.

\section{Patients and methods}

Patients with advanced melanoma who had received RT after ipilimumab progression were eligible for analysis. Radiotherapy was available upon physician request for patients who failed ipilimumab therapy and for whom no other therapeutic options were available.

\section{Results}

21 out of 95 patients treated with ipilimumab in the Italian EAP were eligible for the analysis. The median age was of 58 years (range 21-77); The progression free survival (PFS) from ipilimumab treatment was 4 months (range 3-6), while the time from the end of treatment with ipilimumab and RT was of 5 months (range 4-8).

"Istituto Nazionale Tumori Fondazione "G. Pascale", Naples, Italy

Full list of author information is available at the end of the article
RT was performed on brain in 13 (62\%) patients: 8 were treated with whole-brain RT and 5 patients with stereotactic RT. Other RT treatment included bone, metastatic distant lymph nodes, sub-cutaneous metastasis, spinal cord metastatis. The median doses of radiation was of $30 \mathrm{~Gy}$ (range 30-50). A local response to RT was detected in 13 patients $(62 \%)$ while 8 patients $(38 \%)$ did not show any local regression. The abscopal response has been detected in 11/21 (52\%) patients: in details, we observed 9 abscopal partial response (42,8\%), 2 abscopal stable disease $(9,6 \%)$, and 10 progression $(47,6 \%)$. The median of occurrence of the abscopal response was of 1 month (range 1-4). The median overall survival (OS) for all the 21 patients was of 13 months (range 6-26). The median OS for patients with and without abscopal responses was respectively of 22.4 months (range 2,5-50,3) and 8,3 months (range 7,6-9.0). 11 (84,6\%) out of 13 patients with local response showed an abscopal effect.

\section{Conclusions}

The RT after ipilimumab treatment may be an option for further potentiate its effect. Local response to RT might be predictive for the abscopal response and outcome. Further studies are warranted in this field to better understand and define the role of RT in combination or sequencing with ipilimumab treatment.

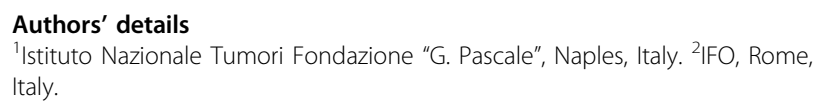


doi:10.1186/2051-1426-1-S1-023

Cite this article as: Grimaldi et al:: The abscopal effect: efficacy of radiotherapy in patients on progression after treatment with

ipilimumab $3 \mathrm{mg} / \mathrm{kg}$. Journal for ImmunoTherapy of Cancer 2013

1(Suppl 1):O23.

Submit your next manuscript to BioMed Central and take full advantage of:

- Convenient online submission

- Thorough peer review

- No space constraints or color figure charges

- Immediate publication on acceptance

- Inclusion in PubMed, CAS, Scopus and Google Scholar

- Research which is freely available for redistribution

Submit your manuscript at 